\title{
sciendo
}

\section{Causality and Modelling in the Sciences: Introduction}

\author{
María Jiménez-Buedo \\ Universidad Nacional de Educación a Distancia \\ Federica Russo \\ University of Amsterdam \\ BIBLID [0873-626X (2017) 47; pp. 423-427] \\ DOI: $10.1515 /$ disp-2017-0013
}

\begin{abstract}
The advantage of examining causality from the perspective of modelling is thus that it puts us naturally closer to the practice of the sciences. This means being able to set up an interdisciplinary dialogue that contrasts and compares modelling practices in different fields, say economics and biology, medicine and statistics, climate change and physics. It also means that it helps philosophers looking for questions that go beyond the narrow 'what-is-causality' or 'what-are-relata' and thus puts causality right at the centre of a complex crossroad: epistemology/ methodology, metaphysics, politics/ethics. This special issue collects nine papers that touch upon various scientific fields, from system biology to medicine to quantum mechanics to economics, and different questions, from explanation and prediction to the role of both true and false assumptions in modelling.
\end{abstract}

\section{Keywords}

Causality, modelling, causal explanation, scientific models.

Traditionally, in philosophy, questions about causality have been typically associated to the nature of the causal relation or of the relata. Thus, for instance, Hume took issue with the 'necessary' character of causal relations and famously thought of causal relata as 'objects'. In contemporary philosophical debates analogue arguments have been put forward by metaphysicians worrying about causality. From David Lewis to John Mackie, from Ned Hall to Jim Woodward, 
broad metaphysical questions have occupied the scene in detriment of other issues more directly related to methodology.

But there have been also gradual changes, partly introduced by the influential Judea Pearl, who developed methods for causal analysis based on algorithmic approaches already widely used in artificial intelligence. This historical reconstruction, though loose and too brief, serves the purpose of introducing the topic of this special issue: causality and modelling. Modelling is no doubt a central part of science, and it has been thoroughly investigated since (at least) Neopositivism, but what is its relation to some of the intricate questions aroud causality? Several recent contributions have shown that (causal) modelling is not 'one thing', but actually many. For instance, in modelling practices it is worth distinguishing between causal inference (finding out what causes what) and causal explanation (explaining how a cause produces its effect). Studying causality from the perspective of modelling also allows us to bring in to the picture the relation between causality and knowledge, or causality and the world. In terms of more classic topics in philosophy of science, we may ask whether, to what extent, or how causal modelling have contributed (or hindered) scientific process or conceptual change. Or how causal models are (or ought to be) used to design policy in socioeconomic of public health contexts. There is also a battery of questions that belongs more naturally to the 'modelling' debate, but that are also tailored to causal modelling, for instance invariance (under intervention), robustness, or exogeneity.

The advantage of examining causality from the perspective of modelling is thus that it puts us naturally closer to the practice of the sciences. This means being able to set up an interdisciplinary dialogue that contrasts and compares modelling practices in different fields, say economics and biology, medicine and statistics, climate change and physics. It also means that it helps philosophers looking for questions that go beyond the narrow 'what-is-causality' or 'whatare-relata' and thus puts causality right at the centre of a complex crossroad: epistemology/methodology, metaphysics, politics/ethics. In fact, our methods concepts, ontologies, and also values are not independent from each other. So, for instance, one could argue that a given causal model suits a biological phenomenon but not a sociological one. Or, one could argue that our standards of evidence 
to establish causal relations affect the usability of said knowledge in policy contexts. In sum, there is a plethora of questions to be addressed about causality and modelling. This special issue collects nine papers that touch upon various scientific fields, from system biology to medicine to quantum mechanics to economics, and different questions, from explanation and prediction to the role of both true and false assumptions in modelling.

In his paper, Jon Williamson argues that objective Bayesian can be useful in the tackling some of the challenges faced by systems medicine, and that arise with the use of big data. Williamson addresses the problems of how to integrate evidence and the problem of how to structure the development of models to then show how the Bayesian approach can help in both.

Veli-Pekka Parkkinen compares the epistemic roles of theoretical models and model organisms in biomedicine. Parkkinen departs, with Levy and Currie (2014), from the idea that animal models share the same broad epistemic role with theoretical models (as surrogate systems providing indirect representation of their targets). However, while Levy and Currie defend that model organism research and theoretical modelling differ in the justification of model-to-target inferences, Parkkinen argues, by means of biological case studies, that the distinctions pointed out by Levy and Currie have to do with the epistemic role or purpose of studying a model, rather than the strategies that are used to validate those models.

In his paper, Dana Matthiessen analyzes the modelling practices of system biologists and defends that contrary to views that causal claims and explanations are rare in this subdiscipline, many models of gene regulatory networks aim at representating causal structures in ways that are compatible with an interventionist conception of causal structure, thus vindicating the applicability of causal interventionist approaches to complex biological systems.

Maria Serban's piece takes issue with Turing patterns, a class of minimal mathematical models that have been used to discover and conceptualize certain abstract features of early biological development. Her paper argues that these minimal mathematical models aid in structuring the epistemic practices of biology by providing precise descriptions of the quantitative relations between various features of the complex systems, generating novel predictions that can 
be compared with experimental data, promoting theory exploration, and acting as constitutive parts of empirically adequate explanations of naturally occurring phenomena, such as biological pattern formation.

Margherita Benzi's paper examines the underlying causal models embedded in the definitions of a controversial medical condition, the Metabolyc Syndrome. Her paper shows how the controversy around the definition is at least partially based on the different causal content of the various definitions. She thus distinguishes among: (1) definitions grounded on associations, (2) definitions presupposing a causal model built upon statistical associations, and (3) definitions grounded on underlying mechanisms.

In their paper, Riet Van Bork, Lisa D. Wijsen , and Mijke Rhemtulla take issue with the Common Factor Model, which models the relations between the observed indicators and the latent variable, and it is typical of many psychological constructs (such as intelligence, as defined by intelligence tests) that are typically unobserved and are therefore measured by observing so-called indicators of the latent construct. In their article the authors argue in favor of interpreting the CFM as a causal model rather than merely a statistical model, in which common factors are only descriptions of the indicators, since, (1) a causal interpretation conforms with most research questions in which the goal is to explain the correlations between indicators rather than merely summarizing them; (2) a causal interpretation of the factor model legitimizes the focus on shared, rather than unique variance of the indicators; and (3) a causal interpretation of the factor model legitimizes the assumption of local independence.

Caterina Marchionni deals with the modelling practices of Economists. In particular, she revisits the debate around the possibility of model-based explanations in economics, where models are riddled with unrealistic assumptions on which the model's results often directly depend. The author reviews critically some of the arguments against the idea that economic models can be explanatory to then focus on the role that non-empirical and empirical strategies play in increasing confidence in the adequacy of a given model-based explanation.

Northcott's piece, in turn, stands against common wisdom regarding the preeminence of explanation over prediction. The author shows, via the case studies of predictive modelling in opinion polling of political elections and the case of weather forecasting, that prediction 
can be the only and more essential fruit of a model, even if that same model cannot explain electoral results or weather outcomes.

Christian de Ronde's paper provides a general account of the causal models which attempt to provide a solution to the measurement problem of Quantum Mechanics and argues that the many interpretations which can be found in the literature can be distinguished through the way they model the measurement process, either in terms of the efficient cause or in terms of the final cause. In contrast to these schemes De Ronde presents a model based on the immanent cause which, he argues, provides an intuitive understanding of the measurement process in QM.

The papers collected in this special issue reflect various ways in which causality and modelling can relate. Some of them have been presented at the conference Causality and Modelling in the Sciences (CaMitS), which took place in the summer of 2015. After the conference an open call for papers had been issued and all submitted paper underwent the usual refereeing process. We are extremely grateful to the 'Causality in the Sciences' steering committee, and all conference participants for making it such an insightful event. We are also grateful to the editors Teresa Marques and Celia Teixeira for their warm welcome to our proposal for a special issue and to Jose Manuel Mestre for his editorial assistance. Last, but not least, we are hugely indebted to all referees for assisting us in the process.

María Jiménez-Buedo Universidad Nacional de Educación a Distancia (UNED) mjbuedo@fsof.uned.es

Federica Russo University of Amsterdam F.Russo@uva.nl 